\title{
The trochanteric double contour is a valuable landmark for assessing femoral offset underestimation on standard radiographs: a retrospective study
}

Stefan Blümel ${ }^{1 \dagger}$, Vincent A. Stadelmann ${ }^{2^{* \dagger}} \mathbb{D}$, Marco Brioschi ${ }^{1}$, Alexander Küffer ${ }^{3}$, Michael Leunig ${ }^{1}$ and Hannes A. Rüdiger ${ }^{1}$

\begin{abstract}
Background: Inaccurate projection on standard pelvic radiographs leads to the underestimation of femoral offset-a critical determinant of postoperative hip function-during total hip arthroplasty (THA) templating. We noted that the posteromedial facet of the greater trochanter and piriformis fossa form a double contour on radiographs, which may be valuable in determining the risk of underestimating femoral offset. We evaluate whether projection errors can be predicted based on the double contour width.

Methods: Plain anteroposterior (AP) pelvic radiographs and magnetic resonance images (MRIs) of 64 adult hips were evaluated retrospectively. Apparent femoral offset, apparent femoral head diameter and double contour widths were evaluated from the radiographs. X-ray projection errors were estimated by comparison to the true neck length measured on MRIs after calibration to the femoral heads. Multivariate analysis with backward elimination was used to detect associations between the double contour width and radiographic projection errors. Femoral offset underestimation below $10 \%$ was considered acceptable for templating.

Results: The narrowest width of the double line between the femoral neck and piriformis fossa is significantly associated with projection error. When double line widths exceed $5 \mathrm{~mm}$, the risk of projection error greater than $10 \%$ is significantly increased compared to narrower double lines, and the acceptability rate for templating drops below 80\% ( $p=0.02)$.

Conclusion: The double contour width is a potential landmark for excluding pelvic AP radiographs unsuitable for THA templating due to inaccurate femoral rotation.
\end{abstract}

Keywords: Total hip arthroplasty, Femoral offset, Templating, Projection error, Double contour, Trochanter major

\footnotetext{
* Correspondence: vincent.stadelmann@kws.ch

†'Stefan Blümel and Vincent Stadelmann contributed equally to this manuscript as first authors.

${ }^{2}$ Department of Teaching, Research and Development, Schulthess Klinik, Lengghalde 2, CH-8008 Zürich, Switzerland

Full list of author information is available at the end of the article
}

C C The Author(s). 2021 Open Access This article is licensed under a Creative Commons Attribution 4.0 International License, which permits use, sharing, adaptation, distribution and reproduction in any medium or format, as long as you give appropriate credit to the original author(s) and the source, provide a link to the Creative Commons licence, and indicate if changes were made. The images or other third party material in this article are included in the article's Creative Commons licence, unless indicated otherwise in a credit line to the material. If material is not included in the article's Creative Commons licence and your intended use is not permitted by statutory regulation or exceeds the permitted use, you will need to obtain permission directly from the copyright holder. To view a copy of this licence, visit http://creativecommons.org/licenses/by/4.0/. The Creative Commons Public Domain Dedication waiver (http://creativecommons.org/publicdomain/zero/1.0/) applies to the data made available in this article, unless otherwise stated in a credit line to the data. 


\section{Background}

The reconstruction of abductor lever arms is critical for hip function after total hip arthroplasty (THA). In clinical practice, femoral offset-defined as the distance between the center of the femoral head and anatomical axis of the proximal femur-is used to simplify the biomechanical analysis of native or prosthetic hip joints and is an important determinant of postoperative hip function [1]. Postoperative changes in femoral offset may lead to altered muscular function that can result in hip abductor muscle insufficiency and pain as well as early prosthesis wear $[2,3]$. Furthermore, loss of femoral offset necessitates increased abductor muscle force to maintain normal gait pattern [4], and a loss exceeding $15 \%$ can result in gait alterations [5].

Assessment of femoral offset is routinely made on plain anteroposterior (AP) radiographs of the pelvis. However, this measurement is regularly underestimated because of incorrect projection. True anatomical hip measurements are made using computed tomography (CT), but this technique is limited in routine clinical work because of the associated radiation exposure levels and high costs. When compared to CT scan measurements, radiographic femoral offset is critically underestimated in as many as $28 \%$ of THA patients [6].

For templating THA on plain radiographs, it is pivotal to assess the projection quality resulting from possible femur malrotation. The lesser trochanter is commonly used as a guide for assessing femoral anteversion, but quantifying rotation with it is imprecise [7, 8]. Another possible landmark to consider is the external obturator footprint: together with the greater trochanter posteromedial facet, they are visible as two contours on AP radiographs [9]. If this easily identifiable double contour is narrow and almost superimposed, the underestimation of femoral offset appears to be minimal. Therefore, our aim was to examine whether the double contour width is predictive of the projection error on plain pelvic AP radiographs. We hypothesized that femoral rotation relative to the $\mathrm{x}$-ray plane may be estimated by measuring the distance between the two contours of the posteromedial trochanteric facet, which may be considered a good predictor of femoral projection.

\section{Methods}

For this observational study, we retrospectively examined radiographs and magnetic resonance images (MRIs) from patients who were aged 16 years and older and consulted our institution for any hip condition between January 2014 until December 2018. This analysis was approved by the Cantonal Ethics Committee of Zurich (KEK-ZH-Nr. 2015-0258) in conjunction with the 1964 Helsinki Declaration and its later amendments or comparable ethical standards.
We included patients who had pelvic AP radiographs and hip joint MRI with femoral antetorsion view, acquired within 1 year of each other. Of 72 patients with complete image documentation, eight were excluded for the following reasons: open growth plates $(n=1)$, poor image quality $(n=1)$, severe hip dysplasia $(n=2)$, Morbus Perthes $(n=2)$ or a prior hip surgery $(n=2)$. Our final cohort included 64 patients with a mean age of 30 years (Table 1). All patients provided written informed consent to use their clinical data for research and publication purposes.

\section{Hip image evaluations}

Plain pelvic radiographs were acquired at $81 \mathrm{kV} / 40 \mathrm{mAs}$ according to our clinical protocol with a slight internal rotation of the legs of $10-15^{\circ}$ to compensate for the femoral antetorsion, and the radiographic cassette was placed two fingerbreadth over the iliac crest. On these radiographs, the double contour is the lateral part of the intertrochanteric crest at the posteromedial facet of the greater trochanter and projection of the convex cortex of the piriformis fossa (Fig. 1). We focused on measuring the double contour separation width and apparent femoral neck length. The latter was chosen because unlike femoral offset, femoral neck length is independent of femoral axis that is difficult to assess accurately due to the limited field on pelvic radiographs, CT scans and MRIs.

Multiple anatomical variants of the double contour can be observed. The most common variant appears as an S-shaped line and one less curved line, another less common appears as superimposed lines and the last, more rare variant, is seen as two curvy lines (Fig. 2a-d). To account for these variants, we defined the width of the double contour (including line thickness) at three levels perpendicular to the femoral axis: D1 at the femoral neck level, D3 as the maximum thickness of the double line and D2 as the minimum thickness between D1 and D3 (Fig. 2e-h).

For each pelvic AP radiograph, the double contour widths (D1, D2, D3), apparent femoral neck axis length $\left(\mathrm{L}_{\mathrm{N}} *\right)$ and apparent femoral head diameter $\left(\mathrm{D}_{\mathrm{H}}{ }^{*}\right.$ for calibration) were measured manually with the measurement tools of the DICOM viewer (JiveX 5.0.2.2, VISUS Health

Table 1 Patient data

\begin{tabular}{ll}
\hline & Data $^{*}$ \\
\hline Patients/hips & $64 / 64$ \\
Age (years) & $29.9 \pm 11.1(16-77)$ \\
Sex (male/female) & $26 / 38$ \\
Side (right/left) & $37 / 27$ \\
Time between MRI and radiograph (days) & $15 \pm 75(-186-197)$ \\
\hline
\end{tabular}

*Values presented either as the number or mean \pm standard deviation (range) 


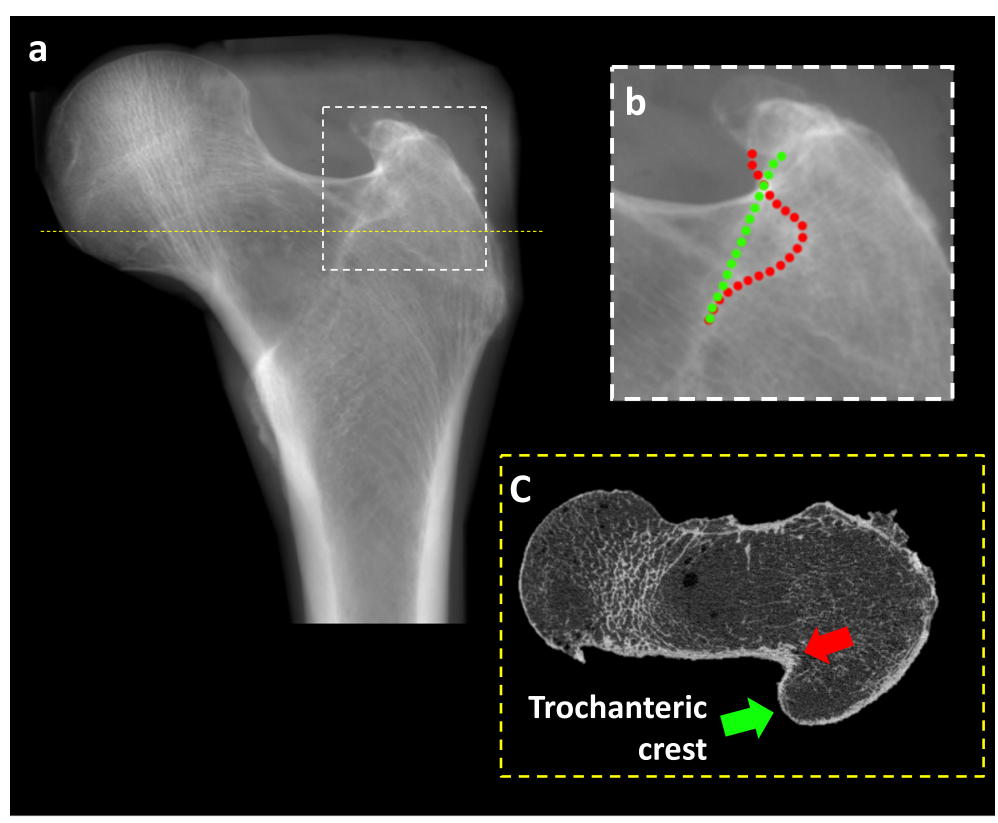

d

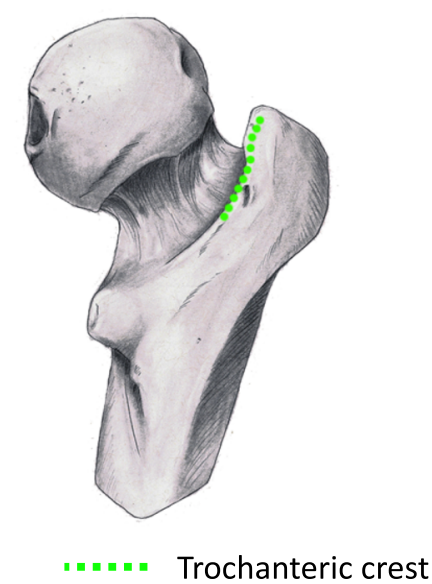

e

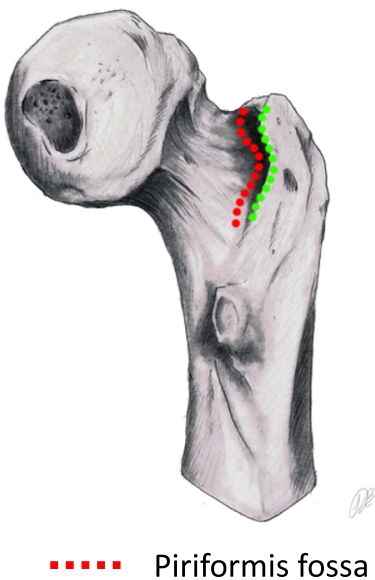

Fig. 1 The double contour highlighted within the boxed area (a) on the pelvic AP radiograph comprises the most common aspect of one Sshaped line (outlined by red dots) and the second line with minimal curvature (green dots) (b). On a high-resolution CT scan (c), the origin of the contours are identified as the trochanteric crest (green arrow) and piriformis fossa (red arrow). In profile view, both lines are superimposed (d) and as the femur rotates, the two lines appear as separate elements (e)

IT GmbH, Bochum, Germany). For $\mathrm{L}_{\mathrm{N}}{ }^{*}$, we measured the full bone length spanning the cortical surface of the femoral head to the opposing cortical surface along the femoral neck axis. $D_{H^{*}}$ was measured by fitting a circle to the articular surface (Fig. 3a). To evaluate the interrater reliability of these measures, twenty randomly selected images were evaluated independently by three physicians. Intra-rater reliability was determined based on one rater reading the same images twice with 4 weeks between assessments.

MRIs of the hip joint with a view of the distal femur for antetorsion measurement were acquired as described by Sutter et al. [1]. True femoral neck axis length $\left(\mathrm{L}_{\mathrm{N}}\right)$ and true femoral head diameter $\left(D_{H}\right)$ were also measured on MRIs of each hip in our cohort to calculate the underestimation in femoral neck length projection on the corresponding plain radiographs. Using multiplanar reconstruction (3D $\mathrm{MPR}$ ) in the DICOM viewer, the MRIs were manually aligned parallel to the plane through both the femoral neck axis and the axis through the proximal femoral diaphysis. $\mathrm{D}_{\mathrm{H}}$ and $\mathrm{L}_{\mathrm{N}}$ were then measured using the same landmarks as those observed on the plain radiographs (Fig. 3b). Femoral antetorsion was also quantified on MRIs as described by Botser et al. [10].

Two main factors affect length measurements on a radiograph: (1) geometric magnification resulting from 


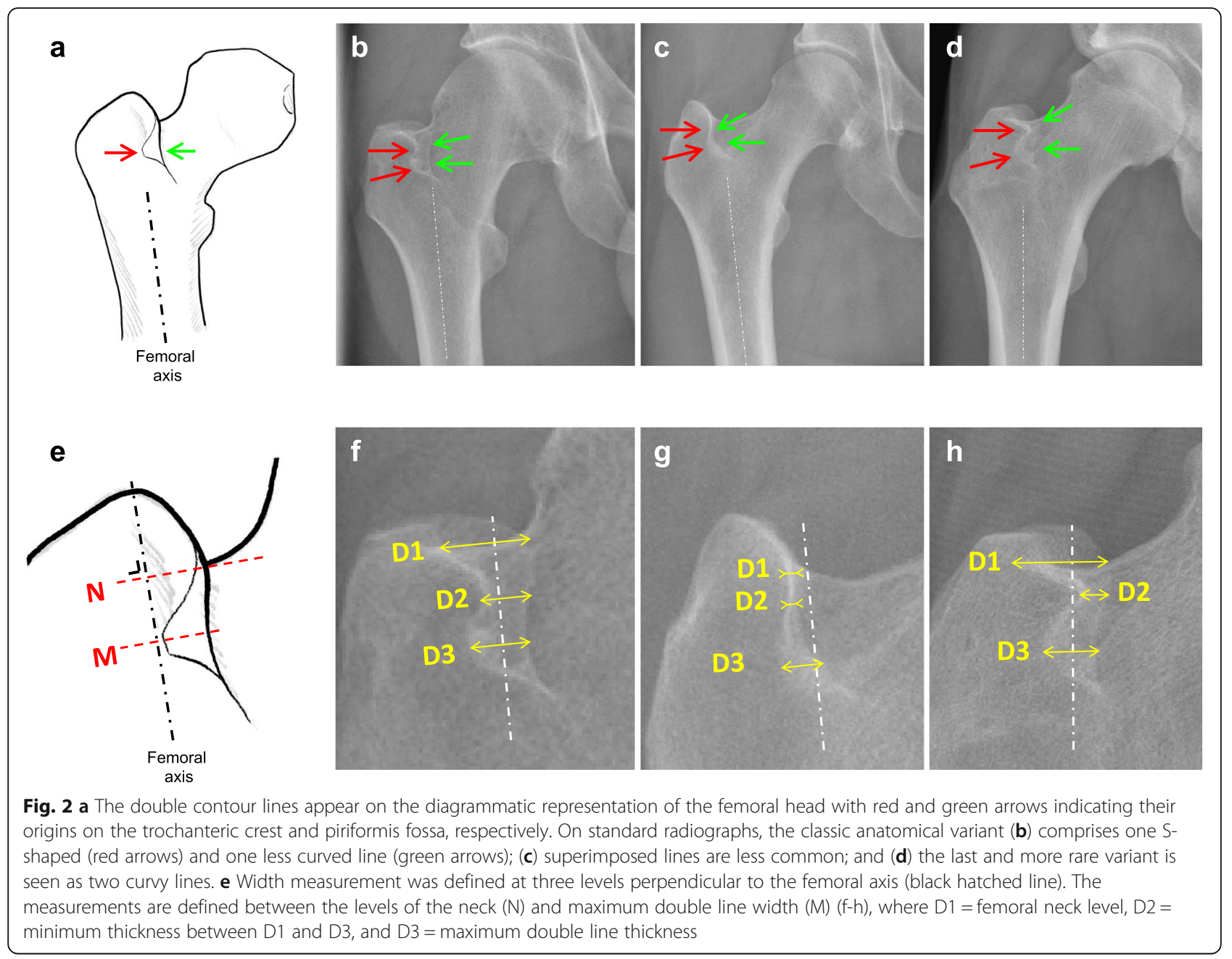

the distance between the object (patient) and film (camera) and (2) geometric distortion (projection error) resulting from the angle between the object and film relative to the $\mathrm{x}$-ray beam [9]. We used the femoral head diameter to correct for magnification with a correction factor $\left(D_{H^{\prime}} / D_{H_{*}}\right)$ : the apparent neck length corrected for magnification $\left(L_{N} \#\right)$ was calculated as ,

$$
L_{N^{*}}=L_{N^{*}} \times D_{H} / D_{H^{*}}
$$

Projection error, as indicated by $E_{\text {proj }}$ was calculated from the difference between the apparent femoral neck length (corrected for magnification) and true femoral neck length,

$$
\text { Eproj }=\left(L_{N}-L_{N^{\sharp}}\right) / L_{N}
$$

\section{Statistical analysis}

All analyses were carried out using R [11]. Normality of the data was confirmed with Q-Q plots and Shapiro-Wilk tests. The data was modelled with generalised multivariate regression analyses using backward elimination to determine whether there was an independent association between the calculated projection errors and anatomical parameters with the significance level set to $p<0.05$. Intraclass correlation coefficients (ICCs) were calculated using the irr package [12] to determine the intra- and inter-rater reliabilities of all image measurements. In addition, double contour widths were categorised by intervals of $2 \mathrm{~mm}$ and an "acceptability rate" (AR) was defined as the fraction of radiographs with a projection error $<10 \%$ within a defined interval. Distributions of ARs were compared with Chisquare proportion tests. Study data were collected and managed using REDCap electronic data capture tools hosted at our clinic $[13,14]$.

\section{Results}

The anatomical measurements of antetorsion, neck length, head diameter and double contour width were normally distributed for our patient cohort (Table 2); all measurements, except antetorsion, were significantly larger for males $(p \leq 0.0001)$. The mean calculated projection error was $2 \%$ higher for female patients $(p=0.024)$. 

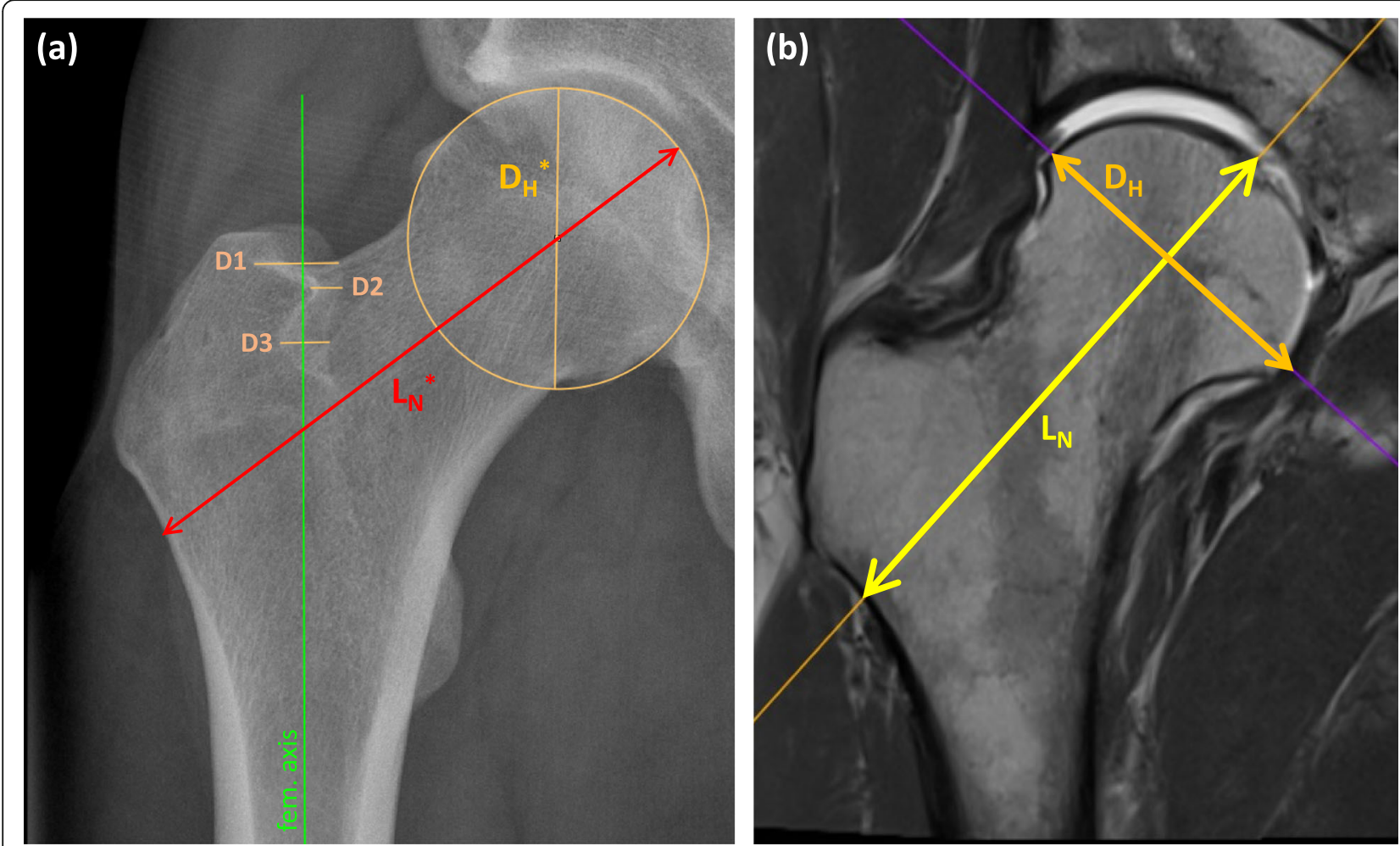

Fig. 3 a The apparent femoral neck length, $L_{N}^{*}$, was measured on the anteroposterior radiograph as the full bone length spanning the cortical surface of the femoral head to the opposing cortical surface along the femoral neck axis. Apparent femoral head diameter $\left(D_{H}{ }^{*}\right)$ was measured by fitting a circle to the articular surface. $\mathbf{b}$ True femoral neck length and femoral head diameter were measured on the magnetic resonance image in the sagittal plane of the femur and compared to the corresponding radiographic measurements

Intraclass correlation coefficients (ICC) for inter-rater reliability were $0.99,0.98$ and 0.97 for D1, D2 and D3, respectively. For intra-rater reliability, the respective ICCs for D1, D2 and D3 were 0.99, 0.93 and 0.97. Interrater reliability for $\mathrm{D}_{\mathrm{H}}$ and $\mathrm{L}_{\mathrm{N}}$ were 0.95 and 0.99 respectively.

Based on the backward stepwise multivariate regression analysis, antetorsion, gender, D1 and D3 were not associated with projection errors. However, there were associations between projection error and the D2 double contour width (Fig. 4a), age (Fig. 4b) and hip side (right) (Fig. 4c); the respective mean ( \pm standard deviation [SD]) effect sizes were $0.57 \pm 0.24$ (95\% confidence interval [CI]: 0.08, 1.06),$-0.59 \pm 0.24$ (95\%CI: - 1.08, - 0.11), and $-1.06 \pm 0.48$ (95\%CI: $2.01,-0.10)$. For the former, the effect size of 0.57 indicates an increase in projection error of $0.57 \%$ for each $1 \mathrm{~mm}$ change in $\mathrm{D} 2$.

Table 2 Distribution of anatomical measurements made on plain pelvic radiographs according to sex

\begin{tabular}{|c|c|c|c|c|c|}
\hline & \multicolumn{2}{|l|}{ Male } & \multicolumn{2}{|l|}{ Female } & \multirow[t]{2}{*}{$P$-value } \\
\hline & Mean \pm SD & Range & Mean \pm SD & Range & \\
\hline Antetorsion $\left(^{\circ}\right)$ & $8.0 \pm 10.0$ & $(-14.5,26)$ & $10.6 \pm 9.3$ & $(-8.7,35)$ & 0.3200 \\
\hline Full neck length (mm) & $100.8 \pm 7.0$ & $(88.4,119)$ & $88 \pm 4.7$ & $(78.8,99)$ & $<0.0001$ \\
\hline Femoral head diameter (mm) & $48.4 \pm 2.7$ & $(43.6,53)$ & $41.7 \pm 2.4$ & $(37.7,48)$ & $<0.0001$ \\
\hline D1 (mm) & $11.7 \pm 4.5$ & $(2.3,20)$ & $5.8 \pm 3.1$ & $(1.5,15)$ & $<0.0001$ \\
\hline D2 (mm) & $6.6 \pm 2.9$ & $(1.4,13)$ & $3.5 \pm 2.0$ & $(0.6,8)$ & $<0.0001$ \\
\hline D3 (mm) & $10.3 \pm 3.6$ & $(4.3,18)$ & $6.6 \pm 2.4$ & $(2.4,12)$ & 0.0001 \\
\hline Projection error (\%) & $19.5 \pm 2.7$ & $(15.3,27)$ & $21.4 \pm 4.1$ & $(13.2,30)$ & 0.0240 \\
\hline
\end{tabular}

\section{SD standard deviation}

$P$-value $=$ Shapiro-Wilk test $p$-value

$\mathrm{D}=$ width of the double contour (including line thickness) at three levels perpendicular to the femoral axis, where D1 lies at the femoral neck level, D3 indicates the maximum thickness of the double line and D2 indicates the minimum thickness between D1 and D3 


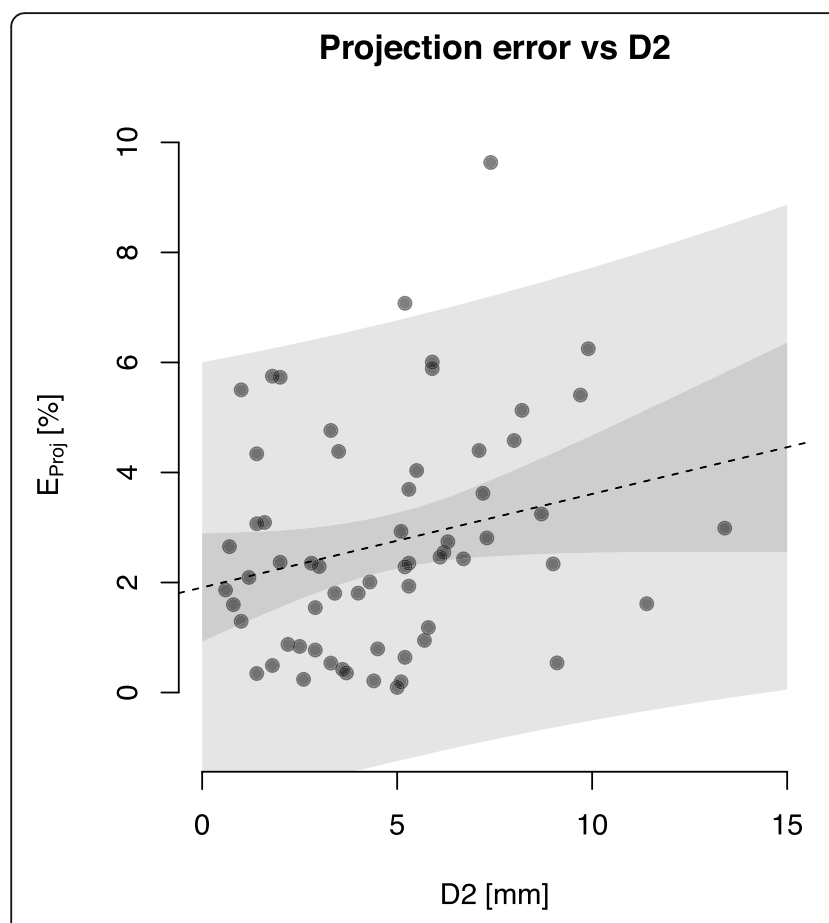

Proj. error vs Side

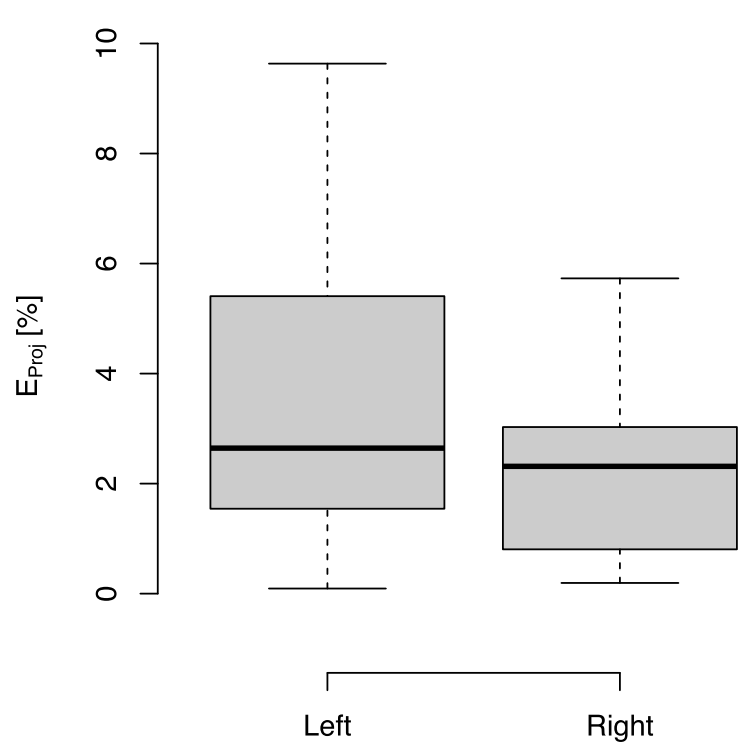

Proj. error vs Age

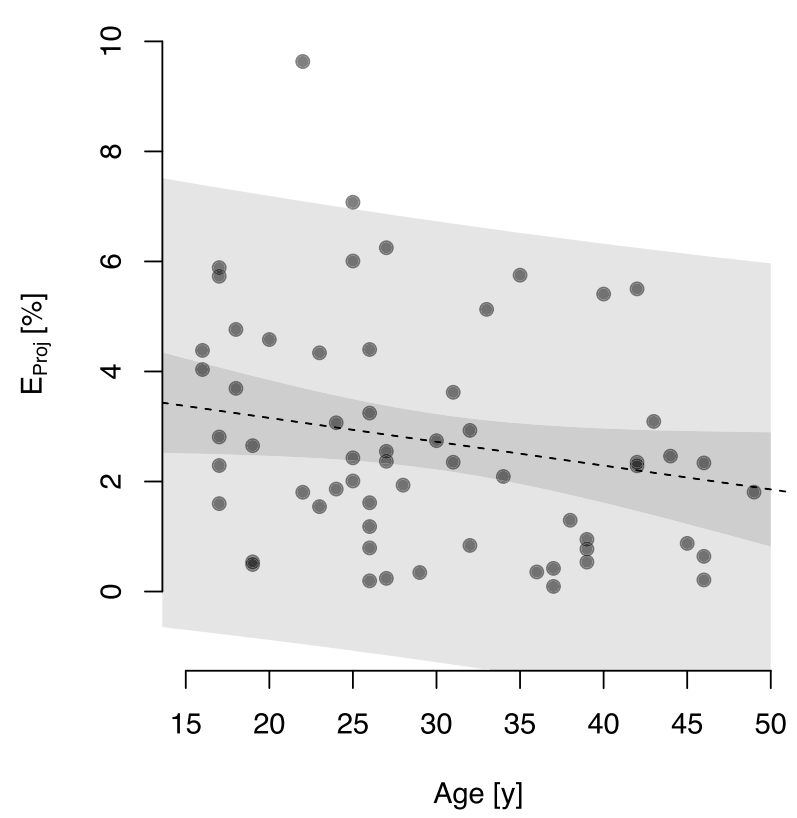

Acceptable rate vs D2

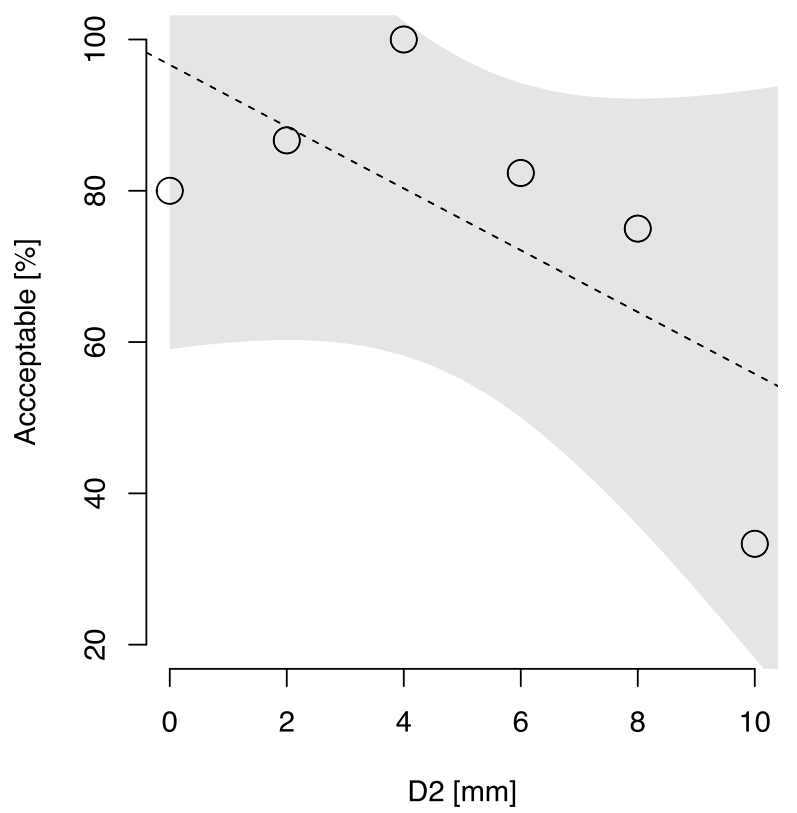

Fig. 4 Plots generated with R showing the correlations between (a) the D2 double contour width, (b) age and (c) hip side with projection error. The rate of acceptability of radiographs drops below $80 \%$ for D2 widths higher than $5 \mathrm{~mm}$ (d) 
Lastly, the AR was negatively associated with D2 width $(-4 \%$ per $\mathrm{mm}, p=0.02)$. In particular, AR dropped below $80 \%$ for D2 values larger than $5 \mathrm{~mm}$ (Fig. $4 \mathrm{~d}$ ).

\section{Discussion}

From our retrospectively analysed imaging data, we wanted to examine whether the double contour created by projection of the greater trochanter posteromedial facet is predictive of the projection error of femoral offset on plain pelvic AP radiographs. Although we found weak correlations between various parameters related to the posteromedial facet and femoral neck projection error, there was a significant trend showing that wider double contours are associated with higher femoral projection errors. Based on our regression modelling, the D2 double contour width-the narrowest width between the contours-correlated with the projection error of femoral offset.

Accurate reconstruction of femoral offset is mandatory in THA. Most surgeons use plain pelvic AP radiographs for templating and postoperative quality control, which often leads to inconsistencies in the correct projection of offset due to inaccurate femoral positioning between the $x$-ray beam and film. Internal rotation is also often limited in the presence of osteoarthritis, which adds to the difficulties in achieving correct femoral positioning. A reliable predictor for the accuracy of femoral projection on plain pelvic radiographs such as the D2 double contour width would naturally aid the surgeon in daily clinical practice.

When compared to $\mathrm{CT}$ scan measurements, radiographic femoral offset is underestimated by over $5 \mathrm{~mm}$ (or $~ 15 \%$ of neck length) in $28 \%$ of THA patients [6]. Based on the fact that a loss of femoral offset by $15 \%$ is associated with gait alterations [5], we considered a femoral projection error (leading to an underestimation of femoral offset) of less than $10 \%$ as acceptable. Based on this definition, the proportion of acceptable radiographs dropped below $80 \%$ when D2 was wider than $5 \mathrm{~mm}$. The D1 (width at the level of the femoral neck) and D3 (width at the level of the piriformis fossa) double contour widths were less reliable predictors of femoral malrotation; this might be explained by the variable shape of the double contour, which could affect D1 and D3 to a greater extent than D2.

Being female and younger were associated with a higher offset error. We speculate that for these patients who are generally smaller, the measurement errors are proportionally larger. We also observed a small but significant difference in offset error between right and left hips. This fits with findings from Sutter et al who reported higher antetorsion in the left legs of both healthy and femoroacetabular impingement patients [15]. This asymmetry could potentially favour projection errors on one side since patient positioning protocols are not sidespecific. Assessment of bilateral imaging in large cohorts would be needed to confirm or reject any of these conjectures.

Interestingly, there was no statistical association between antetorsion and projection error, although in theory, under perfectly standardized patient positioning, this association should exist. This reflects how perfect positioning is hard to achieve in real life, and further justify the need for a simple projection quality control procedure.

The inter- and intra-rater reliability of our double contour width measurements were very high. All examiners used the same assessment protocol, which provided a clear guideline instructing them on how to evaluate the double contour. We believe the evaluation of the double contour is easy and reproducible, and recommend its' use in assessing the reliability of a radiograph before measuring femoral offset.

There are several limitations of our study. The study design is retrospective in nature and its power is limited only to patients with a medical indication (most likely affecting the hip joint) who required MRI and radiograph assessments. Most, if not all, of our cohort included patients with hip problems, especially at a young age. We cannot consider our patients as healthy candidates regarding joint function, since many had an anatomical issue as the cause of their dysfunction; our study cohort is not representative of the general population. There can be anatomical variants that do not present the associations observed in our cohort, but a much larger cohort would be needed to identify these variants. The regression parameters would differ with another patient cohort, nonetheless, we assume our results are still highly relevant for THA patients, since we selected patients who consulted for hip problems. Further evaluation of the double contour in a cohort without hip disorders as an indication for the acquisition of an MRI/ $\mathrm{CT}$ and radiograph would be helpful to test our study hypothesis in a healthy population.

\section{Conclusion}

Our data indicate that if the minimal width of the trochanteric double contour between the level of the lateral femoral neck and piriformis fossa exceeds $5 \mathrm{~mm}$ on radiographs from patients with a hip disorder, the risk of underestimating femoral offset by more than $10 \%$ is significantly greater compared to narrower double contours. On this basis, the trochanteric double contour is a potential landmark for excluding pelvic AP radiographs unsuitable for THA templating. Using this particular landmark could greatly assist lower extremity surgeons in discerning between those radiographs that are suitable or not during the routine preparation for THA. 


\section{Abbreviations}

AP: Anteroposterior; AR: Acceptability rate; Cl: Confidence interval; $C T$ : Computed tomography; D: Double contour width (where, for example, D1 = double contour width at femoral neck level); $D_{\mu}$ : True femoral head diameter; $D_{H}{ }^{*}$ : Apparent femoral head diameter; ICC: Intraclass correlation coefficients; $L_{N}$ : True femoral neck axis length; $L_{N}{ }^{*}$ : Apparent femoral neck axis length; MRI: Magnetic resonance image; SD: Standard deviation; THA: Total hip arthroplasty

\section{Acknowledgements}

The authors wish to thank PD Dr. med. Reto Sutter of the Balgrist University Hospital Zürich for his advices, Ursula Eberli and Lukas Kamer of the AO Research Institute for sharing a high-resolution CT scan of a cadaveric femur, Melissa Wilhelmi at Schulthess Clinic for manuscript editing and preparation, and Ana Zimmermann and Marion Claudet for the MRI alignment reliability readouts. We gratefully acknowledge Daniela Biechele for the anatomical artwork.

\section{Authors' contributions}

HAR was the initiator of this analysis. HAR, VAS and SB were involved in the study design. Study was conducted by HAR and VAS; and data collection was undertaken by $\mathrm{SB}, \mathrm{MB}, \mathrm{AK}$, and ML. VAS performed the data analysis and $S B, H A R$ and VAS the data interpretation. Preparation of the manuscript was done by SB, HAR and VAS. All authors have read, revised, and approved the final manuscript.

\section{Funding}

Support for this research was provided by the non-profit Wilhelm Schulthess Foundation. The funding body had no role in the design, execution, analyses, interpretation of the data, or decision to submit results of this study.

\section{Availability of data and materials}

The datasets during and/or analyzed during the current study are available from the corresponding author on reasonable request.

\section{Declarations}

Ethics approval and consent to participate

Institutional Review Board/Ethics Committee Approval was granted by the Cantonal Ethics Committee of Zurich, Stampfenbachstrasse 121/Postfach, 8090 Zurich, Switzerland. Approval for analyses of the local clinical data (KEKZH-Nr. 2015-0258) was granted on 23. June 2015.

All patients provided written informed consent to use their clinical data for research purposes.

\section{Consent for publication}

All patients provided written informed consent to use their clinical data for publication purposes.

\section{Competing interests}

The authors declare that they have no competing interests.

\section{Author details}

'Department of Hip and Knee Surgery, Schulthess Klinik, Zürich, Switzerland. ${ }^{2}$ Department of Teaching, Research and Development, Schulthess Klinik, Lengghalde 2, CH-8008 Zürich, Switzerland. ${ }^{3}$ Department of Neurosurgery, Schulthess Klinik, Zürich, Switzerland.

Received: 15 July 2020 Accepted: 2 March 2021

Published online: 29 March 2021

\section{References}

1. Flecher X, Ollivier M, Argenson JN. Lower limb length and offset in total hip arthroplasty. Orthop Traumatol Surg Res. 2016;102:S9-S20.

2. De Fine $M$, Romagnoli M, Toscano A, Bondi A, Nanni M, Zaffagnini. Is there a role for femoral offset restoration during total hip arthroplasty? A systematic review. Orthop Traumatol Surg Res. 2017;103:349-55.

3. Little NJ, Busch CA, Gallagher JA, Rorabeck CH, Bourne RB. Acetabular polyethylene wear and acetabular inclination and femoral offset. Clin Orthop Relat Res. 2009;467:2895-900.
4. Rüdiger HA, Guillemin M, Latypova A, Terrier A. Effect of changes of femoral offset on abductor and joint reaction forces in total hip arthroplasty. Arch Orthop Trauma Surg. 2017;137:1579-85.

5. Sariali E, Klouche S, Mouttet A, Pascal-Moussellard H. The effect of femoral offset modification on gait after total hip arthroplasty. Acta Orthop. 2014;85: $123-7$.

6. Lecerf G, Fessy MH, Philippot R, Massin P, Giraud F, Flecher X, et al. Femoral offset: anatomical concept, definition, assessment, implications for preoperative templating and hip arthroplasty. Orthop Traumatol Surg Res. 2009;95:210-9.

7. Yun HH, Yoon JR, Yang JH, Song SY, Park SB, Lee JW. A validation study for estimation of femoral anteversion using the posterior lesser trochanter line: an analysis of computed tomography measurement. J Arthroplast. 2013;28: 1776-80.

8. Worlicek MC, Wörner ML, Craiovan BS, Zeman F, Grifka J, Renkawitz TF, et al. Lesser trochanter size on plain anteroposterior radiographs correlates with native femoral anteversion. J Arthroplast. 2017;32:2892-7.

9. Rüdiger HA, Fritz B, Impellizzeri FM, Leunig M, Pfiffmann CW, Sutter S. The external obturator footprint as a landmark in total hip arthroplasty through a direct anterior approach: a CT-based analysis. Hip Int. 2019;29:96-101.

10. Botser IB, Ozoude GC, Martin DE, Siddiqi AJ, Sivaraja K, Domb BG. Femoral anteversion in the hip: comparison of measurement by computed tomography, magnetic resonance imaging, and physical examination. Arthroscopy. 2012;28:619-27.

11. R Core Team. R: A language and environment for statistical computing [Internet]. Vienna, Austria; 2018. Available from: https://www.R-project.org/

12. Shrout $P E$, Fleiss JL. Intraclass correlations: uses in assessing rater reliability. Psychol Bull. 1979;86:420-8.

13. Harris PA, Taylor R, Thielke R, Payne J, Gonzalez N, Conde JG. Research electronic data capture (REDCap)-a metadata-driven methodology and workflow process for providing translational research informatics support. J Biomed Inform. 2009;42:377-81.

14. Harris PA, Taylor R, Minor BL, Elliott V, Fernandez M, O'Neal L, et al. The REDCap consortium: building an international community of software platform partners. J Biomed Inform. 2019;95:103208.

15. Sutter R, Dietrich TJ, Zingg PO, Pfirrmann CWA. Femoral antetorsion: comparing asymptomatic volunteers and patients with femoroacetabular impingement. Radiology. 2012;263:475-83.

\section{Publisher's Note}

Springer Nature remains neutral with regard to jurisdictional claims in published maps and institutional affiliations.
Ready to submit your research? Choose BMC and benefit from:

- fast, convenient online submission

- thorough peer review by experienced researchers in your field

- rapid publication on acceptance

- support for research data, including large and complex data types

- gold Open Access which fosters wider collaboration and increased citations

- maximum visibility for your research: over $100 \mathrm{M}$ website views per year

At $\mathrm{BMC}$, research is always in progress.

Learn more biomedcentral.com/submissions 